\title{
An Adaptive Filter Approach for Development in Performance of Chaotic Sequences
}

\author{
$\mathrm{K}_{\text {Renu }}^{\# 1}$, P Rajesh Kumar ${ }^{* 2}$ \\ "Department of Electronics and Communication Engineering, \\ GITAM University, Visakhapatnam, A. P, India \\ * Department of Electronics and Communication Engineering, \\ Andhra University, Visakhapatnam, A.P, India \\ 1renuengg12@gmail.com \\ ²rajeshauce@gmail.com
}

\begin{abstract}
Pulse compression technique is the active research topic and widely used in various applications which requires sequences that has good correlation properties. In radar and spread spectrum systems random white noise is used that is similar to the sequence called chaotic sequence as it provides auto and cross-correlation properties similar to that of random white noise. The generation of good sequences which are applicable to radar systems are derived from chaotic maps. These sequences provide better performance in range resolution and detection range. The measures of performance are autocorrelation sidelobe peak (ASP), peak sidelobe ratio (PSR). This paper presents a novel method which improves these all performances. The sequence considered here is the four phase codes which are generated from chaotic map equations. Because the properties of these codes are same as that of random four phase codes. In this paper adaptive equalizer using least mean square (LMS) algorithm is adopted because of its simplicity in implementation and slow convergence. By using this novel method LMS algorithm superior performance can be achieved. However, the number of sequences is not limited by the length of the sequence. Simultaneously the improved performance of mean square error is also observed.
\end{abstract}

Keywords- Chaotic maps, Peak Sidelobe Ratio, Autocorrelation Sidelobe Peak, Least Mean Square Algorithm, Mean square error

\section{INTRODUCTION}

Pulse compression technique has wide applications in various fields such as sonar, radar and spread spectrum and multiple access communication. A well-established radar technique is used in order to attain high transmit energy of a long pulse while keeping the range resolution of a short pulse. Pulse compression solves the practical problem of extending the operating range of radar while at the same time maintaining the required range accuracy along with the resolution. It is achieved by modulating the long transmitted pulse which will be correlated with its received reflection.

Pulse compression technique has two different approaches. In the first approach the codes with small sidelobes in their auto correlation pattern are processed. In the second approach non recursive time invariant and recursive variant causal filters are used. In pulse compression technique the echo signal from the target passes through the matched filter and the output of the filter has spike like mainlobe and noise like sidelobes [1], [2]. These sidelobe masks the main lobe of weak target echo signals which are undesirable. The ratio of the peak sidelobe to the peak of the mainlobe is called as PSLR. In order to maximize the signal to noise ratio of the received signal at the output of the matched filter various pulse compressed waveforms have been used which includes linear frequency modulated signal and the nonlinear frequency modulated signal like biphase and polyphase sequences [3]. The radar performance depends on its range resolution which is obtained from the auto correlation pattern.

Earlier it was reported that a binary phase code is generated using chaotic logistic map in order to achieve low PSLR. However peak sidelobe ratio of longer length binary sequences was reported by Linder [4], Rao and Reddy [5]. It was shown that the binary barker sequence of length 13 has a PSLR of $-22.3 \mathrm{~dB}$ [6]. The aperiodic auto correlation function of good binary and ternary sequences is obtained for different length and they have ideal energy efficiency [7], [8].

It is required to minimize sidelobe peak to obtain low value of PSLR and the best codes are generated based on this low peak sidelobe ratio value. The value of PSLR for longer length binary and ternary sequences was obtained where it was observed that there is drastic improvement of PSLR performance [9]. But as the peak sidelobe ratio is not reduced much for longer binary and ternary sequences it became essential to switch over to various phase sequences. J.W.Taylor, generated good quadriphase or fourphase sequences using biphase to quadriphase transformation [10]. However, Turny analyses four phase barker codes [11]. The behaviour of 
Doppler shift for quadriphase sequence is also studied by Taylor and Blinchikoff which is same as biphase sequence but the ambiguity function of fourphase code is different from that of biphase code [12]. It is quite interesting that the performance of four phase sequences is good compared to binary and ternary sequences [13].

In this paper the improvement of peak sidelobe ratio for four phase sequence is described by using adaptive filtering technique. Adaptive filtering is a regulating system which takes the help of recursive algorithms for processing [14]. In order to suppress the sidelobes, an adaptive finite impulse response filter is used next to the matched filter. This adaptive filter is implemented with least mean square algorithm [15], [16]. The design of the filter means optimization of filter coefficient. Designing of filters for suppressing the range sidelobes, results in minimum peak sidelobe value, has been a subject of interest and this is the area where lots of research work is going on. The technique discussed here has advantage over the previous methods used for four phase sequences.

This paper is structured into the following sections. Section II reviews the generation of fourphase code while section III deals with the adaptive filtering technique. The proposed method of design implementation is presented in section IV. The comparison and simulation results are detailed in section V.

\section{Generation of Four Phase Sequence}

The generation of four phase sequence comprises of subpulses which are phased in one of the four states 0,90 , 180, 270 degrees. These phases are equivalent to $+1,+j,-1,-j$. That mean it uses four alphabets for data representation. These sequences of any length is generated by using chaotic map. Chaotic map equation is used to generate a raw sequence of length $\mathrm{N}$. According to the selection of initial value of $\mathrm{x}_{\mathrm{n}}$, a large number of raw sequences are generated. Quantization of raw sequences has carried out by randomly selecting threshold levels $\mathrm{a}, \mathrm{b}$ and $\mathrm{c}$ between minimum and maximum values of $\mathrm{x}$. The values of levels $\mathrm{a}, \mathrm{b}$ and $\mathrm{c}$ vary for different chaotic maps.

\section{A. Chaotic Maps}

Chaos theory is a field of theory in mathematical sub discipline. The various applications of chaos theory are in several disciplines such as computer science, economics, engineering environmental science sociology meteorology, biology philosophy and ecology etc. A small change in initial condition produces a widely diverging outcome for the chaotic system. Chaotic maps follow the behaviour of deterministic chaos meaning hereby that the future characteristics depends fully on initial condition. Chaotic sequences can be generated by using chaotic maps such as Logistic Map, Improved Logistic Map, tent map, cubic Map, Lorenz Map, quadratic Map and Henon Map. Chaotic sequences have the advantage of inherent security and considered for the implementation of CDMA systems.

B. Logistic map

Logistic map in equation (1) is considered as a prototype model that describes the chaotic behaviour of very simple non-linear dynamic equations. The logistic map equation is written as

$$
x_{n+1}=\mu x_{n}\left(1-x_{n}\right), x_{n} \in(0,1) \text { and } \in(0,4)
$$

Where $\mu$ describes the behaviour such as stability, periodicity and chaotic. With different values of $\mu$ the system will oscillate between states. The sequence generated by using logistic map is inherently deterministic. A very small change in $x_{0}$ causes a tremendous difference in the outcome. Below figure shows how the initial condition $x_{0}$ leads to two completely different waveforms.

C. Improved Logistic Map

The equation (2) that describes the improved logistic map is written as

$$
x_{n+1}=1-2\left(x_{n}\right)^{2}, x_{n} \in(-1,1)
$$

Here $x_{0}$ is varying between -1 and 1 . When the initial value $x_{0}$ is beyond this limit, $x_{n}$ tends to infinity as $\mathrm{n}$ tends to infinity.

D. Cubic Map

Cubic map is represented by the equation (3)

$$
x_{n+1}=4\left(x_{n}\right)^{3}-3 x_{n}, x_{n} \in(-1,1)
$$

When $\mathrm{n}$ tend to infinity $x_{0}>1, x_{n}$ also tends to infinity.

E. Quadratic Map

This map is described in equation (4)

$$
x_{n+1}=0.5-4 *\left(x_{n}\right)^{2}, x_{n} \in(-1,1)
$$

The initial value $x_{0}$ varies from -1 to 1 which shows chaotic behaviour. For logistic maps the value of 'a' is selected as half of the mean of the raw sequence, 'b' is chosen as the mean value whereas the value of 'c' is $3 / 40 \mathrm{of}$ maximum value of the raw sequence.

$$
\begin{aligned}
y_{n} & =-1 \text { if } x_{n}<a \\
& =-j \text { if } a \leq x_{n}<b \\
& =+j \text { if } b \leq x_{n}<c \\
= & +1 \text { if } x_{n} \geq c
\end{aligned}
$$


Therefore, a large number of sequences at a given length can be generated by varying the initial condition $x_{0}$. The quantization levels for different chaotic maps are listed in Table 1.

TABLE I Quantization levels of different chaotic maps

\begin{tabular}{|c|c|c|c|}
\hline \multirow{2}{*}{ Chaotic Map } & \multicolumn{3}{|c|}{ Quantization levels } \\
\cline { 2 - 4 } & $\mathrm{a}$ & $\mathrm{b}$ & $\mathrm{c}$ \\
\hline Logistic Map & 0.25 & 0.5 & 0.75 \\
\hline Improved Logistic Map & -0.6 & 0 & 0.6 \\
\hline quadratic Map & -0.25 & 0 & 0.25 \\
\hline Cubic Map & 0.5 & 0 & 0.4 \\
\hline
\end{tabular}

Using all the above mappings four phases sequences of lengths varying from 10 to 5000 were generated. the generation of sequences is fast and simple and also reproducible. The performance of these sequences is measured by using PSLR and ISLR which are obtained from the autocorrelation pattern of the sequences.

Aperiodic autocorrelation pattern is the output of the matched filter The aperiodic autocorrelation of this four phase sequence is determined using equation (5)

$$
r(k)=\sum_{n=0}^{N-1-k} x_{n} x^{*}{ }_{n+k}
$$

Where $\mathrm{N}$ is the length of the sequence and ' $\mathrm{k}$ ' ranging between $-(\mathrm{N}-1)$ to $+(\mathrm{N}-1)$. Due to rapid decay of performance of the correlation some finite length sequences are only utilized. For all the chaotic maps for every length of the sequence good autocorrelation pattern was obtained by varying the initial condition. Considering two different initial points which are very close will give rise to different outcome. In the figure below taking $\mu=4$ and $x_{0}=0.1001$ and $x_{0}=0.1002$ as two initial points it is observed that the logistic map results in two different waveforms after a few iterations.
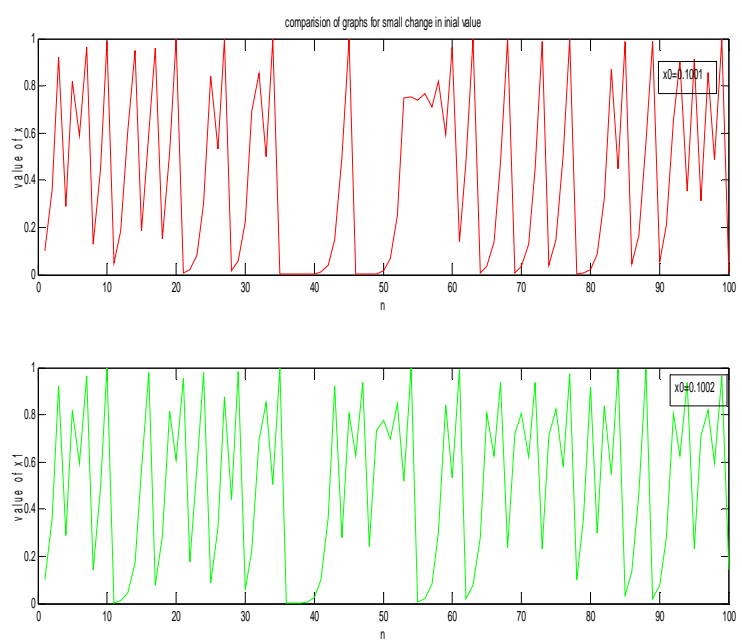

Fig. 1. Variation of Chaotic behaviour of logistic map with initial condition $\mathrm{x}_{0}=0.1001$ and $\mathrm{x}_{0}=0.1002$

Similarly, by a small change in initial condition produces a tremendous change in the output for all the chaotic maps.

Performance Measures: The most commonly used performance measure are PSLR, ISLR \& ASP.

$$
\begin{aligned}
& P S L R=20 * \log 10 \frac{\max r(k)}{r(0)} \\
& I S L R=10 * \log 10\left(2 \frac{\sum_{k=1}^{N-1} r^{2}(k)}{r^{2}(0)}\right) \\
& A S P=\max r(k) \text { where } k \neq 0
\end{aligned}
$$

Therefore, good sequences are selected which are having low PSLR or maximum discrimination factor. And Autocorrelation sidelobe peak which is the maximum value of sidelobe can be measured from ACF must be minimum. Also ISLR will be as low as possible for good sequence. 


\section{III.ADAPTIVE FILTERING}

Adaptive filters are widely used in signal processing and control applications, biomedical applications, radar, sonar and communications. The efficiency of adaptive filters depends on the design technique used and the algorithm that is going to adapt. Adaptive filters are combination of different types of filters such as linear or nonlinear, finite impulse response FIR or infinite impulse response IIR filters and single input or multiple input filter. This adaptation of filter parameters is completely based on minimizing the mean squared error between desired signal and the filter output. Most commonly used adaptive algorithms are Least Mean Square Algorithm, Recursive Least Square Algorithm. Due to simplicity in computation LMS is most widely used in the design implementation of adaptive filters. However, RLS provides high convergence speed compared to LMS but has computational complexity.

An adequate PSLR and ASP can be achieved by using a sidelobe reduction filter after the matched filter such as adaptive filter. Adaptive filters are digital filter that uses feedback as error signal to optimize the transfer function. The basic feature of adaptive filtering includes an input vector ' $\mathrm{x}$ ' and desired response 'd' and an estimated error 'e' which controls the coefficients of adaptive filter. But the coefficients of filter vary from one code to another and it also depends on the filter length. Adaptive filters detect time varying potentials and track the dynamic changes of the signals. The behaviour of these filters changes according to their input signal. The sidelobes can be reduced either by designing a mismatched filter from codes or by employing additional weights after matched filter using optimization algorithm such as Least Mean Square algorithm. There are many other algorithms like Recursive Least Square algorithm (RLS) and modified RLS algorithm. Adaptive filters find various application in signal prediction, noise cancellation etc. The optimum filter weights can be achieved by updating the filter weights.

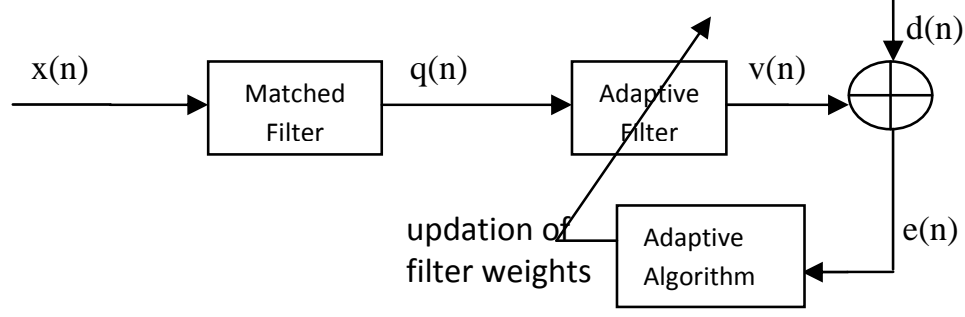

Fig.2. Adaptive filter block diagram

\section{IV.LEASt MEAN Square Algorithm}

This LMS algorithm is a widely used in various applications such as radar systems due to its advantages. The advantages of LMS algorithm includes the following.

- $\quad$ Stable and robust performance on different signal conditions

- Slow convergence

- Simplicity in implementation

The optimum filter weights can be achieved by updating the filter weights in such a manner to converge to optimum. Here assuming a small weight or zero weight and then at each step the weights are updated by finding the gradient of mean square error. This mean square error is a quadratic function filter weights. By ascending or descending down the mean square error provides optimal weights of LMS. The equations of the filter weights for LMS algorithm are as follow.

An input vector $X(n)=[x(n), x(n-1) \ldots x(n-p+1)]^{T}$

Error estimation $e(n)=d(n)-X^{T}(n) * W(n)$

Adaptation of tap-weight vector $W(n+1)=W(n)+2 * \mu * X(n) * e(n)$

Filter output is $y(n)=W^{T}(n) * X(n)$

Where $W(n)$ is the weight vector of adaptive filter at ' $n$ ', $e(n)$ is error signal, $X(n)$ is the input signal vector at ' $n$ ', $d(n)$ is the desired signal, ' $\mu$ ' is the step size which has fixed here and $\mathrm{p}$ is the order of the filter.

In this paper the output of the matched filter is given as input to adaptive filter in order to reduce the autocorrelation peak of the sidelobe level which results in improve in the peak to sidelobe ratio. Here adaptive filter chosen is LMS adaptive filter and the algorithm used to minimize autocorrelation sidelobe level is LMS algorithm.

Although the generation of four phase sequences using different chaotic mapping is easy but all sequences are not appropriate for radar pulse compression. Hence the search for good sequences has done with a small change in initial condition in each mapping methods with and without Least Mean Square Adaptive filter. The best sequences are chosen based on the performance measures defined by equation (6), (7) \& (8). And the results are tabulated for Peak Sidelobe Ratio based on the performance of Autocorrelation Sidelobe Peak and integrated sidelobe ratio obtained for best four phase sequences at different lengths in each chaotic map before and after implementing Least Mean Square algorithm. 


\section{Behaviour of Mean square error}

One of the property of LMS algorithm mean square error. The behaviour of mean square error depends mainly on the step size parameter ' $\mu$ '. The value of ' $\mu$ ' governed the speed of convergence of the algorithm. Generally, a low value of step size is required that results in minimum mean square error value. Hence the selection of this parameter is an important task so that it will give minimum value of mean square error.

\section{Simulation Results}

Simulation has performed by using MATLAB software and the performance is analysed for four phase codes of different length sequence. Figure (3) and figure (4) shows the comparison of Normalized Autocorrelation pattern of logistic map sequence of length 1000 with and without adaptive filter.

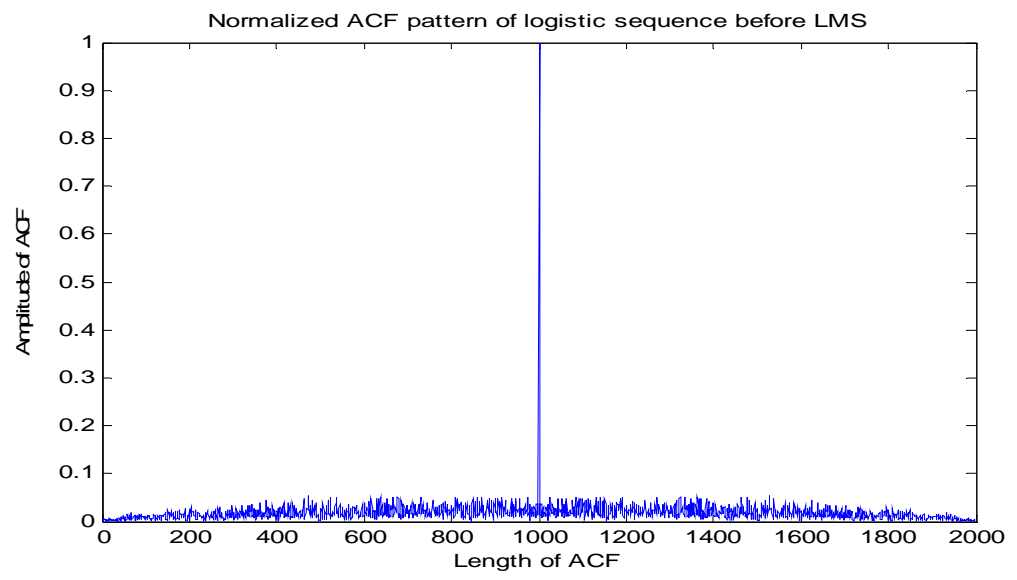

Fig.3 Normalized ACF Pattern of Logistic Sequence of length 1000 without LMS Adaptive Filter

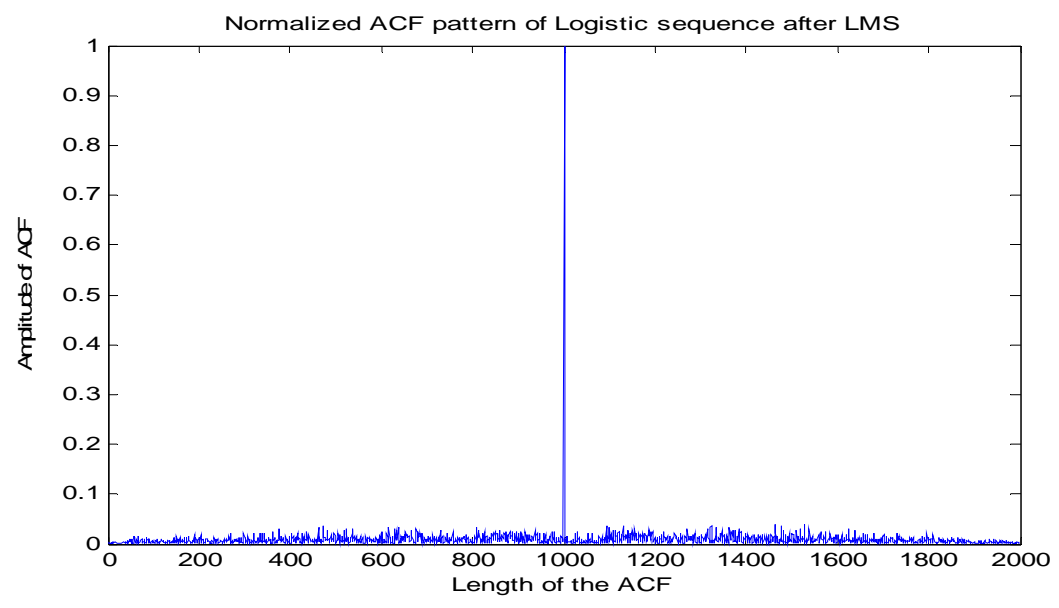

Fig.4 Normalized ACF Pattern of Logistic Sequence of length 1000 with LMS Adaptive Filter

From the above figures it is observed that the Autocorrelation sidelobe peak (ASP) decreases with increase of length. By adapting LMS filter ASP still reduces to a very small value compared to without LMS which causes PSLR to increase.

The autocorrelation pattern of different length of sequence is analysed and the performance results in terms of Peak Sidelobe Ratio, Autocorrelation Sidelobe peak are tabulated for different chaotic maps in table II, III, IV and V. The PSLR is calculated from the equation (6). 
TABLEII Comparison of PSLR\& ASP of Logistic Map Sequence with \& without LMS Adaptive filter

\begin{tabular}{|c|c|c|c|c|}
\hline \multirow{2}{*}{$\begin{array}{l}\text { Length of the } \\
\text { sequence }\end{array}$} & \multicolumn{2}{|c|}{ Logistic Map without LMS } & \multicolumn{2}{c|}{ Logistic Map with LMS } \\
\cline { 2 - 5 } & PSLR & ASP & PSLR & ASP \\
\hline 20 & -16.9897 & 0.1414 & -20.9041 & 0.0901 \\
\hline 30 & -18.4030 & 0.1202 & -20.9452 & 0.0897 \\
\hline 40 & -17.8915 & 0.1275 & -22.0895 & 0.0786 \\
\hline 50 & -18.2974 & 0.1217 & -20.7550 & 0.0917 \\
\hline 60 & -18.4030 & 0.1202 & -22.5696 & 0.0744 \\
\hline 70 & -18.8402 & 0.1143 & -22.7522 & 0.0728 \\
\hline 80 & -18.9237 & 0.1132 & -23.6136 & 0.0660 \\
\hline 90 & -19.2171 & 0.1094 & -20.6714 & 0.0926 \\
\hline 100 & -19.4692 & 0.1063 & -23.2072 & 0.0691 \\
\hline 200 & -21.1776 & 0.0873 & -25.3362 & 0.0541 \\
\hline 300 & -22.5529 & 0.0745 & -26.5432 & 0.0471 \\
\hline 400 & -22.6660 & 0.0736 & -26.3176 & 0.0483 \\
\hline 500 & -23.3348 & 0.0681 & -26.9295 & 0.0450 \\
\hline 600 & -23.8604 & 0.0641 & -26.8464 & 0.0455 \\
\hline 700 & -24.2302 & 0.0614 & -26.0457 & 0.0499 \\
\hline 800 & -24.8293 & 0.0574 & -28.1852 & 0.0390 \\
\hline 900 & -25.1037 & 0.0556 & -28.5746 & 0.0373 \\
\hline 1000 & -25.3820 & 0.0538 & -28.0365 & 0.0396 \\
\hline 2000 & -27.9156 & 0.0402 & -31.0486 & 0.0280 \\
\hline 3000 & -28.9491 & 0.0357 & -29.9156 & 0.0319 \\
\hline 4000 & -30.0390 & 0.0315 & -32.7509 & 0.0230 \\
\hline 5000 & -30.6168 & 0.0295 & -32.6713 & 0.0233 \\
\hline
\end{tabular}

TABLE III Comparison of PSLR\& ASP of Improved Logistic Map Sequence with \& without LMS

\begin{tabular}{|c|c|c|c|c|}
\hline \multirow[t]{2}{*}{$\begin{array}{l}\text { Sequence } \\
\text { Length }\end{array}$} & \multicolumn{2}{|c|}{$\begin{array}{c}\text { Improved Logistic Map } \\
\text { without LMS }\end{array}$} & \multicolumn{2}{|c|}{$\begin{array}{c}\text { Improved Logistic Map } \\
\text { with LMS }\end{array}$} \\
\hline & PSLR & ASP & PSLR & ASP \\
\hline 20 & -16.9897 & 0.1414 & -24.9549 & 0.0565 \\
\hline 30 & -18.4030 & 0.1202 & -20.9452 & 0.0897 \\
\hline 40 & -18.0618 & 0.1250 & -19.2514 & 0.1090 \\
\hline 50 & -18.4164 & 0.1200 & -20.5422 & 0.0939 \\
\hline 60 & -19.0309 & 0.1118 & -22.7541 & 0.0728 \\
\hline 70 & -19.0487 & 0.1116 & -23.2206 & 0.0690 \\
\hline 80 & -19.0309 & 0.1118 & -22.3510 & 0.0763 \\
\hline 90 & -19.5424 & 0.1054 & -24.2675 & 0.0612 \\
\hline 100 & -20.1323 & 0.0985 & -23.0796 & 0.0701 \\
\hline 200 & -21.0100 & 0.0890 & -24.7436 & 0.0579 \\
\hline 300 & -22.0219 & 0.0792 & -25.5071 & 0.0530 \\
\hline 400 & -22.8978 & 0.0716 & -25.7407 & 0.0516 \\
\hline 500 & -23.7018 & 0.0653 & -26.7921 & 0.0458 \\
\hline 600 & -24.2758 & 0.0611 & -27.3132 & 00431 \\
\hline 700 & -24.6051 & 0.0588 & -27.6473 & 0.0415 \\
\hline 800 & -25.0407 & 0.0560 & -28.1943 & 0.0389 \\
\hline 900 & -25.3027 & 0.0543 & -28.5373 & 0.0374 \\
\hline 1000 & -25.6848 & 0.0520 & -28.5387 & 0.0290 \\
\hline 2000 & -27.5845 & 0.0418 & -30.4355 & 0.0223 \\
\hline 3000 & -28.9948 & 0.0355 & -31.5197 & 0.0198 \\
\hline 4000 & -30.0240 & 0.0315 & -31.6582 & 0.0261 \\
\hline 5000 & -31.0688 & 0.0280 & -33.5288 & 0.0211 \\
\hline
\end{tabular}


From the Table. II, III, IV\& V it is observed that PSLR increases with length of the sequence for all types of chaotic maps except cubic map where PSLR is not increasing uniformly. This comparison of PSLR values for different chaotic maps using adaptive filtering technique is shown graphically in the figure 5 . Whereas in figure 6 it is clearly observed that the fourphase sequence performs better than ternary sequence.

TABLE IV Comparison of PSLR\& ASP of Cubic Map Sequence with \& without LMS

\begin{tabular}{|c|c|c|c|c|}
\hline Seq Length & \multicolumn{2}{|c|}{ Cubic Map without LMS } & \multicolumn{2}{c|}{ Cubic Map with LMS } \\
\hline & PSLR & ASP & PSLR & ASP \\
\hline 20 & -16.4782 & 0.1500 & -24.1586 & 0.0620 \\
\hline 30 & -17.5012 & 0.1333 & -20.7538 & 0.0917 \\
\hline 40 & -17.4172 & 0.1275 & -20.7482 & 0.0917 \\
\hline 50 & -17.4473 & 0.1281 & -21.0612 & 0.0885 \\
\hline 60 & -16.9897 & 0.1302 & -21.7976 & 0.0813 \\
\hline 70 & -17.8711 & 0.1152 & -22.7380 & 0.0730 \\
\hline 80 & -178087 & 0.1250 & -20.6565 & 0.0927 \\
\hline 90 & -17.4172 & 0.1160 & -23.4583 & 0.0672 \\
\hline 100 & -17.9588 & 0.1204 & -20.9712 & 0.0894 \\
\hline 200 & -18.5855 & 0.1124 & -22.7877 & 0.0725 \\
\hline 300 & -18.5733 & 0.1202 & -20.6902 & 0.0924 \\
\hline 400 & -17.6875 & 0.1213 & -21.8775 & 0.0806 \\
\hline 500 & -17.8167 & 0.1248 & -22.1883 & 0.0777 \\
\hline 600 & -16.7957 & 0.1246 & -21.8035 & 0.0813 \\
\hline 700 & -16.4296 & 0.1360 & -20.4733 & 0.0947 \\
\hline 800 & -15.9567 & 0.1463 & -19.1931 & 0.1097 \\
\hline 900 & -15.5582 & 0.1536 & -17.9242 & 0.1270 \\
\hline 1000 & -15.5977 & 0.1570 & -18.7229 & 0.1158 \\
\hline 2000 & -14.7630 & 0.1811 & -16.3071 & 0.1530 \\
\hline 3000 & -14.2664 & 0.1827 & -18.8675 & 0.1139 \\
\hline 4000 & -13.9800 & 0.1943 & -15.0911 & 0.1760 \\
\hline 5000 & -13.9749 & 0.2001 & -14.8569 & 0.1808 \\
\hline
\end{tabular}

TABLE V Comparison of PSLR\& ASP of Quadratic Map Sequence with \& without LMS

\begin{tabular}{|c|c|c|c|c|}
\hline $\begin{array}{c}\text { Length of the } \\
\text { Seq }\end{array}$ & \multicolumn{2}{|l|}{ Quadratic Map without LMS } & \multicolumn{2}{l|}{ Quadratic Map with LMS } \\
\hline & PSLR & ASP & PSLR & ASP \\
\hline 20 & -16.9897 & 0.1414 & -20.9041 & 0.0901 \\
\hline 30 & -18.4030 & 0.1202 & -20.9542 & 0.0897 \\
\hline 40 & -17.8915 & 0.1275 & -22.0895 & 0.0786 \\
\hline 50 & -18.2974 & 0.1217 & -20.7550 & 0.0917 \\
\hline 60 & -18.5733 & 0.1179 & -22.4660 & 0.0753 \\
\hline 70 & -18.7722 & 0.1152 & -21.8001 & 0.0813 \\
\hline 90 & -19.5424 & 0.1054 & -23.2221 & 0.0690 \\
\hline 100 & -19.3181 & 0.1082 & -22.6995 & 0.0733 \\
\hline 200 & -21.3966 & 0.0851 & -24.1087 & 0.0623 \\
\hline 300 & -22.1467 & 0.0781 & -25.6241 & 0.0523 \\
\hline 400 & -22.7470 & 0.0729 & -25.6294 & 0.0523 \\
\hline 500 & -23.3161 & 0.0683 & -26.6741 & 0.0464 \\
\hline 600 & -23.8928 & 0.0639 & -27.4642 & 0.0423 \\
\hline 700 & -24.2302 & 0.0614 & -27.4307 & 0.0425 \\
\hline 800 & -24.9210 & 0.0567 & -27.6036 & 0.0417 \\
\hline 900 & -25.0622 & 0.0558 & -28.2327 & 0.0388 \\
\hline 1000 & -25.4166 & 0.0536 & -28.1988 & 0.0389 \\
\hline 2000 & -27.5190 & 0.0421 & -30.4684 & 0.0300 \\
\hline 3000 & -29.0935 & 0.0351 & -31.8358 & 0.0256 \\
\hline 4000 & -29.9361 & 0.0319 & -32.3821 & 0.0240 \\
\hline 5000 & -30.6775 & 0.0292 & -32.8039 & 0.0229 \\
\hline & & & & \\
\hline
\end{tabular}




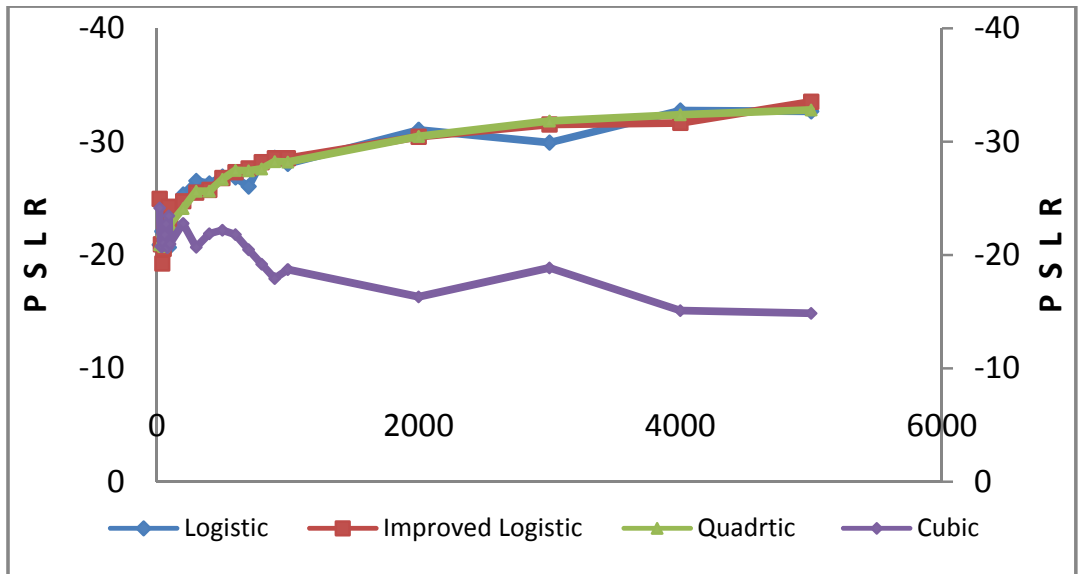

Fig. 5 Comparison of PSLR for Different Chaotic Maps using Adaptive Filters

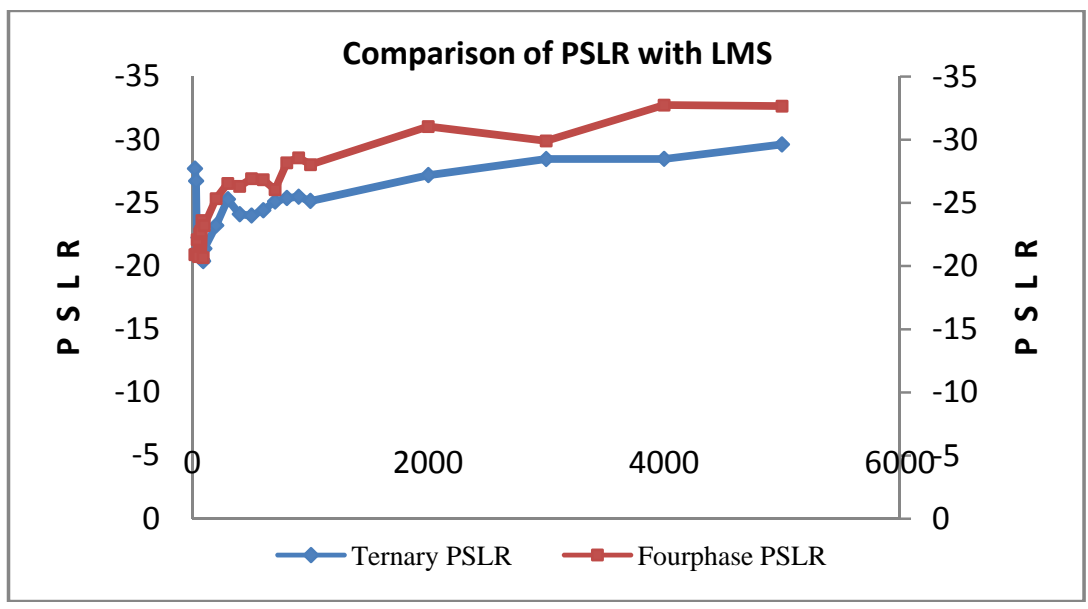

Fig. 6 Comparison of PSLR of ternary and fourphase Logistic sequence for different length

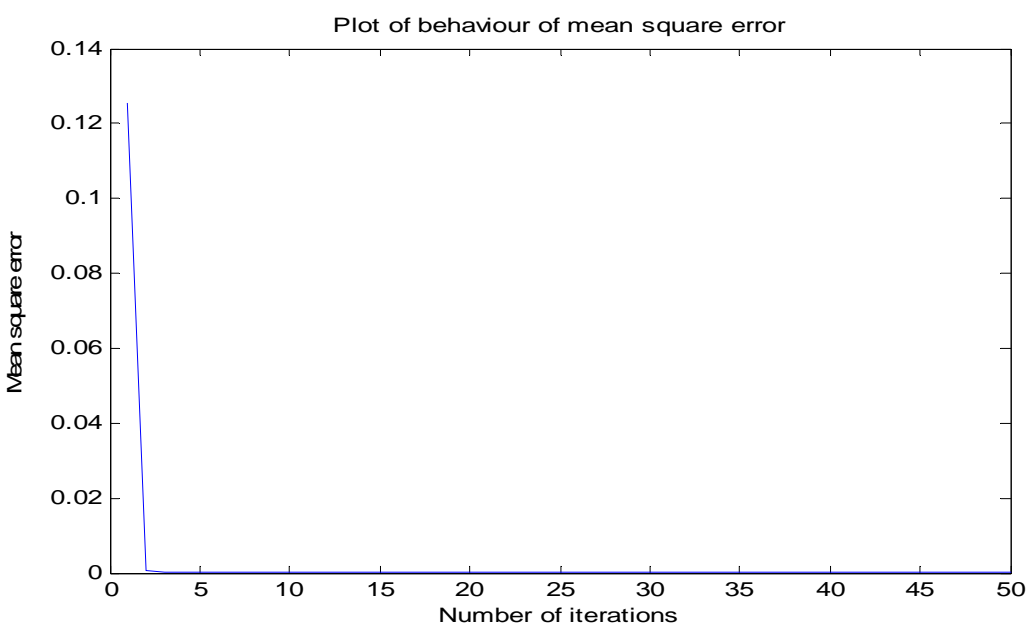

Fig.7 Behaviour of Mean square error for sequence length 1000

The main cause of increase of Peak Sidelobe Ratio in remaining three is only due to decrease in autocorrelation sidelobe peak. It is also observed that by using adaptive filter the PSLR is more negative which means that the value of peak to sidelobe ratio is more. This is because of simultaneous decrease of autocorrelation sidelobe peak with length. It is also analysed that integrated sidelobe ratio, which is the reciprocal of merit factor, decreases with length but by adapting LMS filter its value is comparatively more than that of the value that was obtained previously without using adaptive filter for the same length. Figure 7 shows the behaviour of mean square error which decreases with number of iterations. The number of iterations chosen is 50 with step size parameter 0.05 and filter length 50. The maximum value of MSE obtained is 0.1255 . 


\section{VI.CONCLUSION}

Optimum sequences are generated using logistic, improved logistic, quadratic and cubic maps at various lengths based on performance measures like peak to sidelobe ratio and autocorrelation sidelobe peak. Further improvement in the performance of these sequences is achieved by using adaptive filtering technique. These improvements in the performance results provides various applications in radar and spread spectrum communications. By using adaptive filter with LMS the value of PSLR obtained for length 5000 is less than or equal to $-32.6713 \mathrm{~dB}$ instead of $-30.6168 \mathrm{~dB}$ for logistic map. It is being observed that cubic map shows poor performance compared to other chaotic map discussed here. The proposed algorithm also provides good results in finding minimum value of mean square error with only few iterations for different length of the sequences. The convergence characteristic of LMS depends on minimum mean square error. It is also observed that the maximum value MSE has reduced with length of the sequence. With the above analysis it can be concluded that the adaptive filtering technique with LMS outperforms well in minimizing mean square error with increase in length.

\section{REFERENCES}

[1] Levanon, N. and Mozeson, E.Radar Signals., 2004

[2] Merrill I. Skolnik, 3rd ed, Introduction to Radar System, 2002

[3] COOK, C. E., BERNFIELD, M. Radar Signals - An Introduction to Theory and Application. New York: Academic press, 1967.

[4] Linder. J, "Binary sequences upto length 40 with best possible autocorrelation function”, Electronic Letters, vol.11, no.21, pp. 507508, 1975.

[5] Veerabhadra Rao, K., Umapathy Reddy, "Biphase sequences with low sidelobe autocorrelation function”, IEEE Transactions on Aerospace and Electronic Systems, 1986, vol. 22, no. 2, pp. 128-133.

[6] RAO, B. V., DESHPANDE, A. A. Why the barker sequence bit length does not exceed thirteen. Journal of IETE, Nov-Dec 1998, vol. 34, no. 6, pp. 461-462, 1998

[7] Boehmer, A. M., “Binary pulse compression codes”, IEEE Trans.IT-13,1967, vol no. 2, pp. 156-157.

[8] Xin Wu, Weixian Liu, Lei Zhao and Jeffrey, S., "Chaotic phase code for radar pulse compression”, Proceedings of IEEE National Radar Conference, Atlanta (USA), pp. 279-283, 2001

[9] Seventline. J.B., Elizabeth Rani, D. Raja Rajeswari. K,'Ternary Chaotic Pulse Compression Sequences' Journal of Radio Engineering, Vol.19,No:3, pp.415-420, September 2010.

[10] Taylor, J. W., Jr. and Blinchikoff, H. J., "Quadriphase code-A Radar Pulse Compression Signal with Unique Characteristics”, IEEE Trans. On Aerospace and Electronic systems, vol. 24, n0.2, pp. 156- 170,1988.

[11] Richard, J. Turyn,"Four-Phase Barker Codes”, IEEE Trans. On Inform. Theory, vol. IT-20, n0.3, pp. 366- 371, 1974

[12] Levanon, N. and Freedman, A., “Ambiguity Function of Quadriphase coded Radar Pulse”, IEEE Trans. On Aerospace and Electronic systems, 1989, vol. 25, n0.6, pp. 848-853.

[13] Sridevi. K, J. B. Seventline and Elizabath Rani, D, Four Phase Pulse Compression Sequences Generated using Chaotic Maps", Proceedings of International Journal of Computer Applications, Volume 48-no. 14, pp. 25-30,2012

[14] S. Haykin, 4th ed, "Adaptive Filter Theory”, Pearson Education Asia, 2002.

[15] J.S. Fu, and Xin Wu," Sidelobe suppression using adaptive filtering techniques”, in Proc. CIE International Conference on Radar, Oct. 2001, pp.788 - 791.

[16] Mohammed Umar Shaik and V. Venkata Rao, "Pulse compression techniques of phase coded waveforms in radar", in proceedings of International Journal of Scientific \& Engineering Research, Volume 3, Issue 8, pp.1 - 4, ISSN 2229-5518 August-2012.

\section{AUTHOR PROFILE}

K Renu received her M. Tech degree in Andhra University, Visakhapatnam. At present she is working as Assistant Professor in Department of ECE, GITAM University and pursuing her Ph.D. in Andhra University, Visakhapatnam.

P. Rajesh Kumar received his Ph.D. from Andhra University, Visakhapatnam. He is working as the Head of the Department, Department of ECE, Andhra University, Visakhapatnam. 